Rev. Fac, Med. vet. Zootec. Univ. S. Paulo, 13(2):459-63. 1976.

\title{
CONSIDERACCOES SOBRE OS RAMOS VISCERAIS DA ARTERIA PUDENDA INTERNA DO COELHO (Oryctolagus cuniculus)
}

\begin{abstract}
Antonio Marcos ORSI *
Plinio PINTO E SILVA..

Valêncio José de Mattós CAMPOS "*

Zenon SILVA.

\begin{tabular}{|l|} 
\\
\hline
\end{tabular}

Orsi, A M.: Pintu e Silva. P.: Campos, V.J. De M.: Silva, Z. Consideracóes sobre os ramos riscerais da artéria pudenda interna do coelho (Oryctolagus cuniculus). Rev. Mac. Med. vet. Zootec. Lniv. S. Pallo, 1312):459-63, 1976.

Resumo: Foi realisado estudo anatomico dos ramos viscerais da artéria podenda interna do coelho. Foram utilisados 30 cochos 116 machos e 14 jémeas), adultos, anestesiados com éter etilico, injeludos com contraste na aorta abdominal $127 \mathrm{com}$ látex corado e 3 com massa radiopacal e dissecados sob lupa. Os trés coelhos injetados com massa radiopaca foram previamente radiografados e posteriormente dissecados para comparaçoes com as outras preparaçós. Em resultados süo descritas "origem e a ramescéncia da a. pudenda interna, esta iltima expressada em figuras de porcentagcm relativa.

UnıтеRMos: Artéria pudenda interna*; Coelhos*.
\end{abstract}

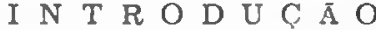

A revisão da literatura especializada não nos apresentou trabalho algum de conduta sistemática, que procurasse es. tabelecer o comportamento arterial dos vasos pélvicos do coelho.

Por outro lado, em tratados especalizados (LESBRE, \& 1923; HYMAN, 2 1942; POPESKO, '; 1954), no capítulo concernente à irrigação arterial pélvica des. ta espécie animal não encontramos referèncias relativas ao comportamento clos ramos viscerais da a. pudenda interna, objetivo deste estudo.

Entre os pequenos mamíferos o comportamento anatômico da a. pudenda interna é descrito no rato (GREENE, 1 1935; MICKWTZ, ' 1956, 1957) e no hams. ter dourado (MICHEL, I 1959). Contudo, cm coelhos não encontramos, na literatura, estudos que tivessem como finaliclade a sistematização arterial da a. pudenda interna, em ambos os sexos, objetivo deste trabalho, que ora apresenta. mos.

- Professor Assistente.

* Professor Titular.

Departamento de Morfologin da Faculdade de Clênclas Mćdicas e Blológricas de Botucatu. 


\section{MATERIAL E MÉTODOS}

No presente trabalho estudamos 30 coelhos (16 machos e 14 fèmeas), adul tos, da raça "Norfolk", sacrificados por inalação de éter etílico. Vinte e sete animais foram injetados, via aorta abdominal, com contraste vascular apropria. do para dissecção sob lupa (látex corddo), e três com contraste radiopaco (massa de Schlesinger, SCHLESINGER, 7 1957).

Após conveniente redução, as preparações foram fixadas em formol a 5\% adicionado de ácido fênico diluído $(0,1 \%)$, para evitar enrijecimento das estruturas anatômicas. As artérias pudendas inter. nas foram abordads após remoção parcil dos ossos ilicos, por corte bilateral dos ramos acetabular do púbis e sinfisário do isquio respectivamente. As artérias ilíaca interna e pudenda foram então dissecadas sob̀ lupa.

As preparaçōes injetadas com con traste radiopaco foram radiografadas afim de comprovar e dirimir possiveis duividas surgidas no decorrer das dissec. çōes e posteriormente, também foram dissecadas.

\section{R E S U L T A D O S}

Apresentamos nossos resultados enfocando a origem e a distribuição da a. pudenda interna em ambos os sexos:

\section{a. Origem}

As observações pertinentes à a. pudenda interna, dissecadas bilateralmente nos 30 animais estu. dados, nos mostraram que esta artéria é a continuação dorsal, direta da a. iliaca interna (Fig. 1).

b. Distribuição:

Em virtude da a. pudenda interna vascularizar estruturas genitais a classificamos em dois grupos, de acordo com o sexo dos animais; dentro de cada grupo encontramos bilateralmen te, modalidades de distribuição da artéria em pauta, de acordo com o comportamento de seus ramos viscerais terminais.

Grupo 1 (Machos: Modalidades A, B, C, D, E - Fig. 2)

Modalidade A: $\mathrm{em} 13$ antímeros $\left(40,63^{0}, \mathrm{u}\right)$, a a pudenda interna terminou bifurcando-se num ramo peniano (for. mando a a. dorsal e a a. profunda do penis) e num ramo retal (a. retal caudal).

Modulidade $B$ : 8 antimeros $(25 \%)$, notamos que a a. pudenda interna findou emitindo um tronco peniano-bulbouretral e um ramo retal.

Modalidade $C$ : em 6 antimeros $(18,75 \%)$, a a. pudenda interna terminou originando um tronco bulbo-uretral-retal c um ramo peniano.

Modalidade $D$ : em 3 antimeros $(9,37 \%)$, a artéria em estudo fornecəu como ramos terminais um tronco pa. niano.bulbo uretral-retal e um ramo retal.

Modalidade $E$ : bilatcralmente, num animal $(6,25 \%)$, a a. pudenda interna cisu origem a um tronco uretro-penianobulbo-uretral e a um ramo retal.

Grupo 2 (Fêmeas: Modalidade A, B, C - Fig. 3).

Modalidade $A: \mathrm{em} \quad 16$ antímeros $(57,15 \%)$, a a. pudenda interna findou, clando origem a um tronco vagino-clitorideano (a. vaginal e a. do clitóris), e a um ramo retal.

Modalidade $B$ : em 7 antimeros (25\%), a a. pudenda interna tərminou formando um ramo vaginal e um ramo retal.

Modalidade $C$ : em 5 antímeros $(17,85 \%)$, a artéria em apraço formou um tronco vagino-retal e um ramo clitorideano. 


\section{DISCUSSÃO}

No que respeita à origem da a. pucienda interna contestamos o relato de LESBRE: (1923), que diz ser esta artéria ramo da a isquiática, pois para nós incontestavelmente, esta artéria é conti. nuaçāo direta da a. ilíaca comum, con. forme nossos resultados. Merece destaque $o$ fato da a pudenda interna percorrer parta de seu trajeto sob a superfície dorsal do corpo do isquio para só a seguir emitir seus colaterais pudendos. Apesar disto, não observamos emissão de ramo algum, isquiático, mas apenas verificamos a sintopia da artéria pudenda interna com o osso ísquio.

Com respeito à origem da a. pu. denda interna em pequenos mamíferos, no hamster dourado, diz MICHEL (1959), que a a. pudenda interna, ramo da a ilíaca interna, forma um arco cerca da terceira vértebra lombar, dirigindo-se a seguir para o arco isquiático. No rato, GREENE1 (1935), relata a origem da a. pudenda interna da superfície dorso.medial da a iliaca interna. No coelho, para nós, é a continuação dorsal, direta da a. iliaca comum.

No que tange à ramanescência visceral da a. pudenda interna do coelho não encontramos citação específica na literatura a não ser em LESBRE: (1923), que de forma genérica, diz que a “a. ilíaca interna emite uma artéria para os órgāos intrapélvicos (reto, próstata, uretra e vagina)", sem a preocupaçāo contudo de denominá-la.

A guisa de comparação, no que respeita a ramescência visceral desta artéria em pequenos mamiferos, diz MICHEL !
(1959), que no hamster dourado macho os ramos viscerais da a. pudenda interna são a a. dorsal do pênis e finos ramos clestinados à glâdula bulbo-uretral, uretra e ânus; no rato, no dizer de GREENE'1 (1935), estes ramos são a a. do pênis do macho, e a a. coo clitóris na fêmea.

Do exposto, pode-se aquilatar que, no que concerne à sistematização arterial da ramescência da a. pudenda interna quer em roedores, ou mesmo especificamente no coelho, objeto de nosso estudo, a literatura falha em agrupar os ramos viscerais em modalidades de distribuiçāo, €scopo de nossa pesquisa.

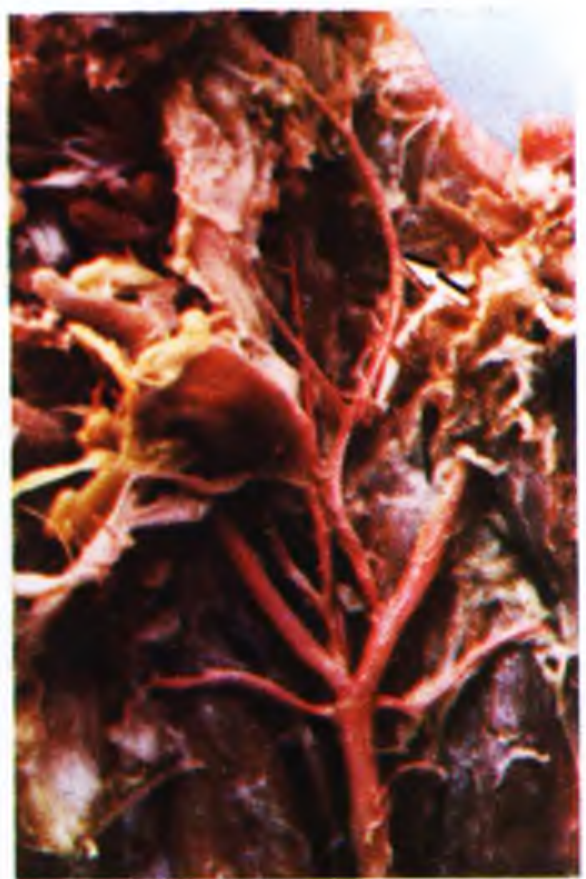

Fig. 1 - A seta assinala a n. pudenda interna do coelho, continuaçio dorsal direta da $\mathbf{n}$ lliaca interna (preparaçāo n.o 7. macho, antimero direito). 


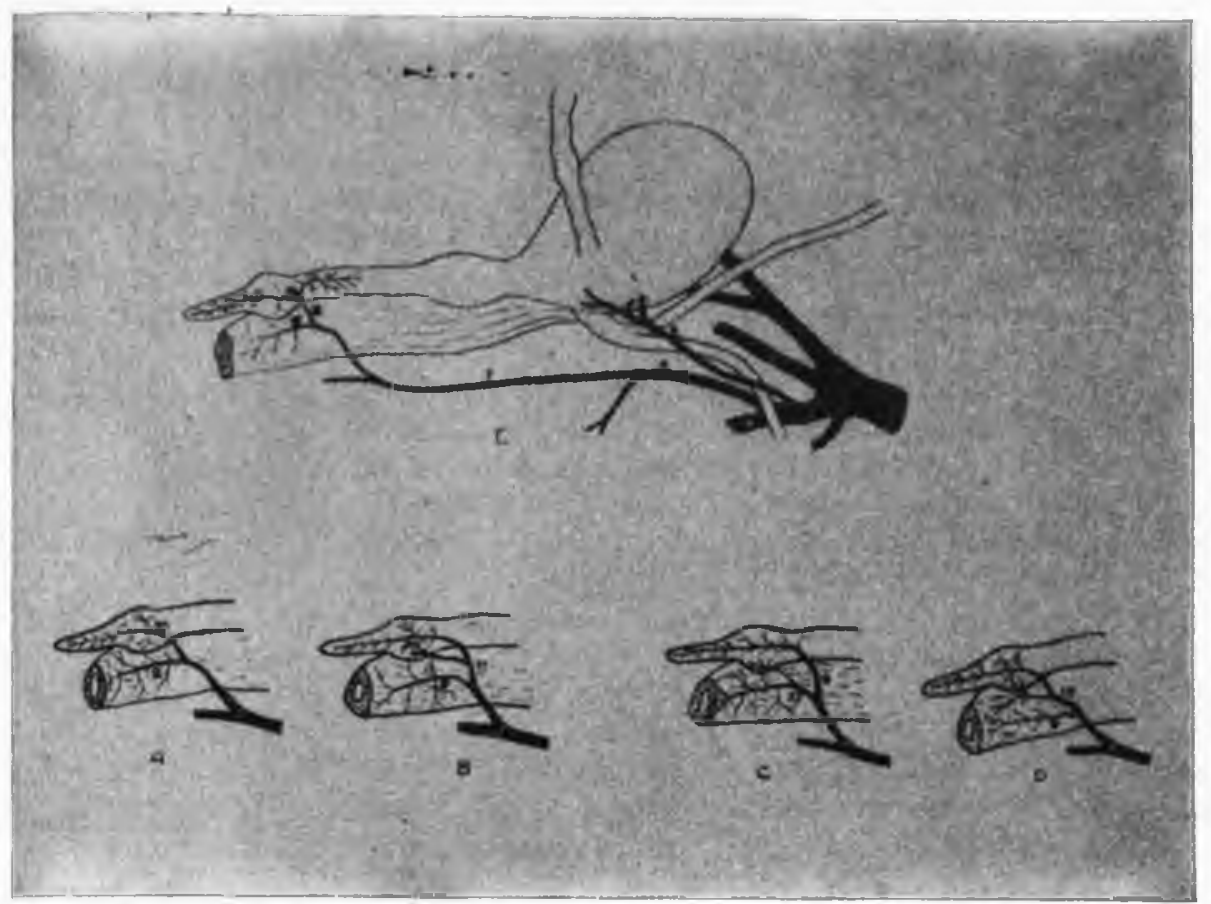

Fig. 2 - Modalidades A. B. C. D. E de distribuíḉo dos ramos viscerais di a. pudenda interna em macho (Oryctolagus cuniculus).

1. Aorta: 2. a. sacra mëdia: 3. a. iliaca comum: 4. a. llíaca interna; 5, a. umbilical; 6. a. Hiaca interna: 7. a. pudenda interna; 8. tronco uretro-penlano-bulbo-uretral; 9. ramo perilano; 11. tronco peniano-bulbo-uretral; 12. tronco bulbo-uretral-retal; 13 . tronco penluno bulbo-uretral-retal.

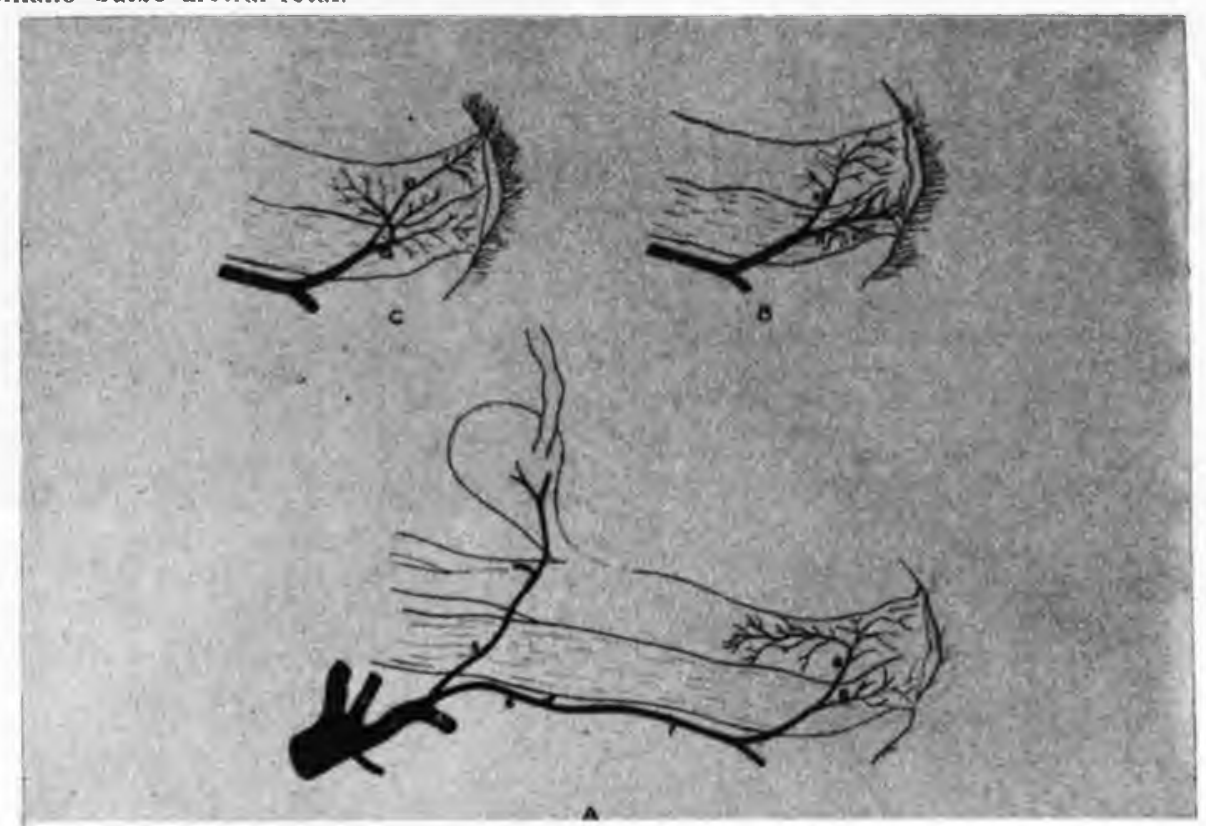

Fig. 3 - Modalidades A. B. C. de distribuiçăo dos ramos viscerais da a. pudenda intern cm fëmea (Aryctolagus cuniculus).

1. Aorta: 2. a. sacra média: 3, a. Jliacn comum: 4. a. lliaca externa: 5. al umbllical: 6. a. Hínca interna: 7. a. pudenda interna; 8. tronco vagino-clitorideano; 9. ramo retal; 10. ramo vaginal; 11 ramo retal; 12 . tronco vagino-retal; 13. ramo clitorideano. 
ORsi. A. MI: PINTo e Silva. P.; Campos, V.J. de M.; Silva, Z. Considerations about the terminal visceral branches of the internal pudendal artery in the rabbit (Oryctolagus cumiculus). Rev. Fuc. Merl. vet Zootec. Iniv. S. Palo, I:3(2):459-63. 1976.

SUMMARY: This paper deals with the analomical study of the visceral branches of the internal pudendal artery in the rabbit.

Thirt! animals (16 males and 14 females), adults, were anaesthetized with cthylic ether, injected with contrasting substance in abdominal aorta (27 animals wth Neoprenc latex and 3 with radio-opac mass) and dissected through stercoscopic microscope. The unimals inject'd with radio-opac mass were radiographed in order to compare with oller preparations.

In results were described origin and branching of this artery. The last result was expressed in relative percentage figures and compared with the rodents arterial disposition.

UNITEMs: Pudendal internal artery"; Rabbits"*.

\section{REFERENCIAS BIBLIOGRAFICAS}

1 - GREENE. E. The anatony of the rat. Trans. Amer. Phil. Soc., 27: 205-6. 1935.

2 - HYMAN. L. H. 'Comparative vertebrate anatomy. Chicago, University of Chlcago Press, 1942, p. 376.

3 - LESBRE, F. S. Prdcis d'anatomic comparés des animaux domestiques. Paris, J. B. Ballliére. 1923. v. 2, p. 383 .

4 - MICHEL. G. 'Die Aufzwelgung der Aorta abdominalis des Syr Gold hamsters mit besonderer Berïcksichtigung der arterlellen Gefassversorgung der minnlichen und welblichen Ge- schlchtsorgane. Anat. Anz. 107: 11125. 1959 .

5 - MICKWITZ npud MICHEL 4. (1959).

6 - POPESKO. P. Allas of thopographical anatomy of the domestic animals. Phlladelphia. Saunders Co., 1954. v. 3. p. 205.

7 - SCHLESINGER. M.J. New radiopaque mass vascular injection. Lab. Invest., 6:1-11. 1957 .

Recebido para publicaçăo em 31-8-76 Aprovado para publlcaçĭo em 13-9-76 